\title{
Desordem temporomandibular e a influência do polimorfismo genético
}

\author{
Temporomandibular disorder and influence of genetic polymorphism
}

Letícia Ladeira Bonato

Membro da equipe do Serviço de Diagnóstico e Orientações a pacientes com DTM da Universidade Federal de Juiz de Fora

VALQUIRIA QUINELATO

Pesquisadora do Instiาtuto Nacional de Traumatologia e Ortopedia (INTO)

José Mauro Granjeiro PhD em Química da Unidade de Pesquisa Clinica - Universidade Federal Fluminense

Priscila Ladeira Casado Pós-doutoranda/ PhD em Morfologia - Unidade de Pesquisa Clinica Universidade Federal Fluminense (UFF)

\begin{abstract}
RESUMO
A desordem temporomandibular (DTM) é uma doença multifatorial que afeta a articulação temporomandibular (ATM), músculos mastigatórios e tecidos adjacentes ocasionando dor crônica na maioria dos pacientes. A progressão da DTM é acompanhada por alterações inflamatórias secundárias determinadas pela interação entre moléculas e células, tais como interleucinas, via Wnt, metaloproteinases e fator de necrose tumoral tipo- $\alpha$. Alterações nos níveis dessas moléculas podem interferir nessa resposta, oferecendo maior proteção ou susceptibilidade ao desenvolvimento da doença. Essa resposta inflamatória é coordenada pela característica genética. Por isso, estudos recentes tentam explorar o padrão genético individual como forma de correlacionar uma predisposição a processos inflamatórios nesta doença multifatorial. Polimorfismos nos genes responsáveis pela resposta inflamatória têm mostrado serem potenciais para futuros procedimentos clínicos em odontologia. Sendo assim, o objetivo desta revisão de literatura foi analisar a correlação entre a característica genética individual e o desenvolvimento de DTM. Baseando-se nos artigos revisados, este estudo demonstrou que a característica genética pode ter correlação com o desenvolvimento de DTM. No entanto, estudos incluindo maior número de pacientes e diagnósticos mais precisos da DTM ainda são necessários para confirmar esta correlação. Acredita-se que futuros métodos diagnósticos e terapêuticos poderão ter como base a característica genética do indivíduo, de acordo com a identificação das regiões polimórficas. Tais métodos poderão propiciar o desenvolvimento de tratamentos padronizados para cada tipo de paciente, identificação de pacientes de risco e protocolos de preservação.
\end{abstract}

Palavras-chave: SINNDROME DA DISFUNÇÃO DA ARTICULAÇÃO TEMPOROMANDIBULAR; POLIMORFISMO GENÉTICO; DOR OROFACIAL. 


\begin{abstract}
Temporomandibular disorder (TMD) is a multifactorial disease that affects the temporomandibular joint (TMJ), the muscles of mastication, and the surrounding tissues, triggering chronic pain as its main consequence. TMD's progress is followed by secondary inflammatory reactions determined by the interaction between molecules and cells, such as interleukins, Wnt pathways, metalloproteinase, and tumor necrosis factors (TNF alpha). Alterations in levels of these molecules can trigger higher protection or susceptibility to the disease. The genetic factor orchestrates the inflammatory reaction. Therefore, recent studies are investigating the individual genetic pattern in order to correlate the predisposition to inflammatory processes in this multifactorial disease. The identification of genetic polymorphism in genes related to inflammatory response may be important to future procedures in dentistry. Thus, the aim of this literature review was to analyze the correlation between the individual genetic characteristics and the development of TMD. Based on reviewed articles, this study showed that genetic characteristics may be related to temporomandibular disorder. However, studies including higher number of patients and a minute diagnosis are necessary in order to confirm such correlation. Futures diagnosis and therapeutic methods should be based on individual genetic characteristics, according to the identification of polymorphic regions. Such methods may lead to the development of standardized treatments for each kind of patient, the identification of at-risk patients and preservation protocols.
\end{abstract}

Keywords: TEMPOROMANDIBULAR JOINT SYNDROME; GENETIC POLYMORPHISM; OROFACIAL PAIN.

\section{INTRODUÇÃO}

As desordens temporomandibulares (DTM) são caracterizadas por um conjunto de condições médicas e dentárias que afetam a articulação temporomandibular (ATM), músculos mastigatórios e tecidos adjacentes, ${ }^{1}$ sendo a condição de dor orofacial crônica a mais enfrentada por cirurgiões-dentistas e outros profissionais da saúde. ${ }^{2}$

A DTM é uma doença multifatorial que pode acometer várias faixas etárias, prevalecendo entre 20 e 45 anos; porém tem-se manifestado com frequência em crianças e adolescentes. ${ }^{3}$ Esta desordem acomete cerca de 1,5 a 2 vezes mais mulheres do que homens, sendo que cerca de $80 \%$ dos indivíduos que buscam tratamento são mulheres. ${ }^{4}$ As razões para esse dimorfismo sexual acentuado estão relacionadas aos hormônios sexuais. ${ }^{5}$

A ATM, como componente do sistema estomatognático, está diretamente relacionada às funções fisiológicas.$^{6,7}$ Aorigem multifatorial da doença, assim como a ausência de diagnóstico definitivo ou abordagens terapêuticas, culmina em um quadro sintomatológico constantemente prolongado, incluindo dor debilitante e queda substancial da qualidade de vida do paciente. ${ }^{6} \mathrm{Na}$ maioria das vezes, os pacientes já consultaram médicos de diversas especialidades na tentativa de solucionar a sintomatologia dolorosa na região dos músculos da cabeça, face, pescoço e ombros. ${ }^{2}$

$\mathrm{Na}$ tentativa de melhor entender a etiopatogenia de inúmeras doenças, recentes estudos têm explorado a característica genética individual. Acredita-se que a associação entre o perfil genético e o desenvolvimento de inúmeras doenças inflamatórias/degenerativas poderá servir de base para futuros métodos diagnósticos e terapêuticos em medicina.

\section{Material e métodos}

Os artigos foram selecionados utilizando as palavras-chave temporomandibular disorder e polymorphism; temporomandibular disorder e genetic factors, na base de dados Pubmed/medline..$^{*}$ Todos os trabalhos que apresentaram correlação com o estudo de polimorfismos em um único nucleotídeo (SNP) foram incluídos, considerando as diferentes populações estudadas em diferentes países. Os critérios de inclusão e exclusão de cada estudo foram respeitados como forma de estudar melhor os dados de genética nas DTMs, tão raramente relatados.

1 www.ncbi.nlm.nih.gov/pubmed. 


\section{REVISÃO DE LITERATURA}

\section{Etiopatogenia e tratamento das DTMs}

Esta desordem tem experimentado, atualmente, mudanças significativas em sua etiologia e tratamento., Durante décadas, acreditou-se que a etiologia da DTM estivesse principalmente relacionada a fatores morfológicos, tais como:alteraçõesnadimensão vertical de oclusão e no posicionamento condilar, luxações mandibulares, desarmonias oclusais e desequilíbrio neuromuscular. Fundamentados neste conceito, inúmeros tratamentos baseados em terapias invasivas e irreversíveis foram adotados nos pacientes, incluindo ajustes oclusais, odontologia restauradora, tratamentos ortodônticos e até mesmo cirurgias. ${ }^{10}$ No entanto, a refratividade da ATM a essas terapias confirma que essa desordem envolve interações muito mais complexas entre mediadores estritamente regulados. ${ }^{11}$

Acredita-se que a dor facial associada com a DTM possui, na maioria das vezes, origem miogênica, sendo que este tipo de dor parece ser mais responsivo a tratamentos conservadores para a DTM. ${ }^{12,13}$

O processo patológico na DTM é caracterizado por deterioração e abrasão da cartilagem articular e espessamento local. Essas mudanças são acompanhadas pela superposição de alterações inflamatórias secundárias. ${ }^{14}$ Os principais mediadores inflamatórios responsáveis por alterações da ATM, cursando ou não com sintomas de dor, são: interleucina IL-6 e IL-1, prostaglandinas (PG), glutamato, estradiol, serotonina (5$\mathrm{HT})$, receptor Fc III (FCRIII), proteínas Wnt (Wnt-5A), proteína S100A12 (Calgranulina C), metaloproteinases (MMP) e TNF- $\alpha .{ }^{15} \mathrm{O}$ $\mathrm{Ca} 2+$ citoplasmático desempenha um papel importante na função normal da célula e seu acúmulo pode ser também um fator-chave que causa fadiga e lesões musculares. ${ }^{4}$
As doenças degenerativas da ATM são também caracterizadas por um desequilíbrio na síntese e degradação de matrizes, mediadas por condrócitos e fibrocondrócitos na cartilagem e fibrocartilagem da ATM, resultando em uma perda progressiva de componentes da matriz extracelular da cartilagem articular e/ou osso subcondral. ${ }^{6}$

No entanto, da mesma forma que em quadros degenerativos de osteoartrose ou de artrite reumatoide sistêmica, doenças inflamatórias que afetam a ATM permanecem como um desafio terapêutico na clínica odontológica, uma vez que não existem tratamentos eficazes até o momento. ${ }^{14}$

\section{Polimorfismo genÉtico}

O polimorfismo genético pode ser caracterizado como a ocorrência de múltiplos alelos em um locus (local ocupado por um gene em um cromossomo), no qual pelo menos um alelo (gene correspondente de dois cromossomos homólogos) aparece em pelo menos $1 \%$ da população. ${ }^{16}$ Loci polimórficos são convencionalmente aqueles para os quais pelo menos $2 \%$ da população é heterozigota. Sendo assim, é considerada a hipótese de que algum fator seletivo tenha atuado sobre aquele sistema genético, levando ao aumento da frequência do alelo anômalo. ${ }^{17}$ Alguns polimorfismos alteram a expressão de um gene e causam um efeito no fenótipo do indivíduo, conferindo suscetibilidade a doenças. ${ }^{16}$ Evidências científicas sugerem que genes específicos podem determinar em qual grau cada resposta imune individual será protetora ou destrutiva. Com as técnicas de DNA recombinante, é possível detectar os polimorfismos que consistem, geralmente, em uma simples troca de base, deleções ou inserções casuais, ou na presença de inúmeras variáveis de cópias repetidas de um determinado fragmento de DNA (repetições em tandem). ${ }^{16}$ 
Estudos tentam correlacionar a presença de polimorfismo genético em genes candidatos ao desenvolvimento de lesões musculares, ósseas, articulares e tendinopatias. $\mathrm{Na}$ DTM, muita importância é dada aos fatores morfológicos, mas a importância relativa dos fatores ambientais em relação aos fatores genéticos para explicar as variabilidades existentes desta desordem permanecem mal compreendidos. ${ }^{18}$

O mecanismo de destruição da ATM é complexo e, como outras doenças complexas, pode envolver polimorfismos genéticos em regiões específicas do DNA que codificam para proteínas que atuam diretamente na degeneração da ATM. ${ }^{19}$ Uma série de diferentes loci genéticos pode estar associada ao desenvolvimento desta patologia, cada um contribuindo com pequenos efeitos que interagem com exposições ambientais, determinando o curso e o resultado da desordem. ${ }^{20}$

De acordo com um estudo realizado em $2011^{21}$ com camundongos mutados, é provável que polimorfismos do gene ANKH (Ankylosis homolog) estejam relacionados com 0 deslocamento de disco da ATM. No entanto, até o momento, poucos estudos em humanos, sem resultados conclusivos, correlacionaram o polimorfismo genético com o desenvolvimento de desordens temporomandibulares (Tabela 1). O primeiro estudo analisando a correlação entre polimorfismo genético e DTM em humanos foi desenvolvido em 2001 por Herken et al. ${ }^{22}$ na população turca. Os autores avaliaram o gene transportador de serotonina (5-HTT) e não encontraram correlação significativa em 159 indivíduos avaliados. No entanto, trabalho posterior de Mutlu et al. ${ }^{23}$ na mesma população encontrou associação entre o gene receptor de serotonina (5-HT2A) e indivíduos com DTM. Diatchenko et al. ${ }^{20}$, em 2006, encontraram associação entre dor na ATM e polimorfismo no gene do receptor adrenérgico tipo 2 na população americana, assim como Kang et al., ${ }^{2}$ em 2007, observaram associação significativa entre polimorfismo no receptor de estrogênio tipo alfa (ERalpha) e mulheres coreanas com DTM.

Atualmente, a via metabólica de folato ${ }^{25}$, a via das metaloproteases (MMPs) ${ }^{15}$ e do Catecol-O-metiltransferase ${ }^{18}$ (COMT) têm sido estudadas na tentativa de encontrar alguma correlação com DTM, apresentando resultados promissores.

\section{Discussão}

ADTMédescrita pelaAssociaçãoAmericana de Dor Orofacial como um termo coletivo que envolve alterações clínicas dos músculos mastigatórios, articulação temporomandibular (ATM), estruturas associadas, ou ambas, ${ }^{30}$ encontrando-se entre as mais comuns condições de dor orofacial de origem não dental. ${ }^{31} \mathrm{Em}$ virtude de sua complexa e multifatorial etiopatogenia, 2,31,32 a desordem apresenta-se muitas vezes com grande variação sintomatológica interindividual. ${ }^{31}$ Por isso, inúmeros estudos têm sido realizados na tentativa de que sejam mais bem definidos os mecanismos predisponentes, causais e perpetuantes desta patologia, ${ }^{29}$ na tentativa de que um número cada vez menor de casos seja insubmisso às terapias para DTM. Portadores de DTM crônica apresentam algum grau de impacto da dor em suas vidas, especialmente nas atividades do trabalho, da escola, no sono e no apetite/alimentação. ${ }^{33} \mathrm{O}$ tratamento dessa disfunção relaciona-se diretamente à melhoria da qualidade de vida do paciente, sendo que a correlação entre causa e efeito é constantemente objeto de investigação. ${ }^{34}$

Baseados neste conceito, a investigação entre a possível associação entre variantes alélicas e a desordem temporomandibular tem aumentado consideravelmente. ${ }^{10}$ Atualmente, acredita-se que fatores genéticos possam desempenhar um papel na determinação 
Tabela 1 - Estudos correlacionando polimorfismo genético e DTM em humanos.

\begin{tabular}{|c|c|c|c|c|c|}
\hline AUTOR & ANO & $\begin{array}{c}\text { AMOSTRA } \\
\text { (INDIVÍDUOS) }\end{array}$ & POPULAÇÃO & POLIMORFISMO & RESULTADO \\
\hline Herken et al. ${ }^{22}$ & 2001 & 159 & Turcos & 5-HTT & $\begin{array}{l}\text { Não foi encontrada associação } \\
\text { entre o polimorfismo e dor na ATM. }\end{array}$ \\
\hline Mutlu et al. ${ }^{23}$ & 2004 & 117 & Turcos & $\begin{array}{l}\text { 5-HT2A Parte inferior } \\
\text { do formulário }\end{array}$ & $\begin{array}{l}\text { O polimorfismo do gene } \\
\text { T102C possivelmente esteja envolvido } \\
\text { na etiologia da DTM. }\end{array}$ \\
\hline $\begin{array}{l}\text { Diatchenko et } \\
\text { al. }{ }^{20}\end{array}$ & 2006 & 202 & $\begin{array}{l}\text { Americanos } \\
\text { (EUA) }\end{array}$ & ADRB2 & $\begin{array}{l}\text { O polimorfismo, incluindo o estudo de } \\
\text { haplótipos para o gene ADRB2, aumentou } \\
\text { a vulnerabilidade à dor oriunda de DTM. }\end{array}$ \\
\hline Kang $^{24}$ & 2007 & 174 & Coreanos & $\begin{array}{l}\text { ERalpha; Pvu II and } \\
\text { Xba I }\end{array}$ & $\begin{array}{l}\text { Encontrada possível associação } \\
\text { com a susceptibilidade da dor } \\
\text { em mulheres portadoras de DTM. }\end{array}$ \\
\hline Ojima et al. ${ }^{26}$ & 2007 & 36 & Japoneses & $\begin{array}{l}\text { Transportador de } \\
\text { serotonina }\end{array}$ & $\begin{array}{l}\text { Um aumento significativo de alelos longos } \\
(\mathrm{L} \text { e } \mathrm{XL} \text { ) foi encontrado nos pacientes com } \\
\text { DTM em relação ao grupo controle. }\end{array}$ \\
\hline Slade et al. ${ }^{27}$ & 2007 & 171 & Australianos & СОMT & $\begin{array}{l}\text { Não há influência dos efeitos do } \\
\text { haplótipo COMT no risco de DTM. }\end{array}$ \\
\hline Kim et al. ${ }^{28}$ & 2010 & 138 & Coreanos & $\begin{array}{l}\text { ERalpha; Pvu II and } \\
\text { Xba I }\end{array}$ & $\begin{array}{l}\text { O haplótipo não foi confirmado } \\
\text { como um marcador importante } \\
\text { para o desenvolvimento de DTM. }\end{array}$ \\
\hline $\begin{array}{l}\text { Tchivileva } \\
\text { et al. }{ }^{29}\end{array}$ & 2010 & 40 & $\begin{array}{l}\text { Ameri- } \\
\text { canos } \\
\text { (EUA) - } \\
\text { Caucasianos } \\
\end{array}$ & $\begin{array}{l}\text { Catecol-O- } \\
\text { metiltransferase } \\
(\mathrm{COMT})\end{array}$ & $\begin{array}{l}\text { Haplótipos COMT podem identificar } \\
\text { um subgrupo de pacientes com } \\
\text { DTM que irão se beneficiar no } \\
\text { tratamento com propranolol. }\end{array}$ \\
\hline $\begin{array}{l}\text { Aneiros- } \\
\text { Guerrero } \\
\text { et al. }{ }^{25}\end{array}$ & 2011 & 229 & Espanhóis & $\begin{array}{l}\text { Enzimas da via } \\
\text { metabólica do folato; } \\
\text { DRD4; GSTM1 }\end{array}$ & $\begin{array}{l}\text { Polimorfismos genéticos relacionados } \\
\text { com a via metabólica do folato, estresse } \\
\text { oxidativo inflamatório e as respostas } \\
\text { à neurotransmissão da dor foram } \\
\text { significativamente associados à DTM. }\end{array}$ \\
\hline Planelo et al. ${ }^{19}$ & 2011 & 232 & Brasileiros & $\begin{array}{l}\text { MMP1 1G/2G; MMP3 } \\
\text { 5A/6A; MMP9 C/T }\end{array}$ & $\begin{array}{l}\text { Associação entre o genótipo 2G/2G } \\
\text { MMP1 e degeneração da ATM; não } \\
\text { houve associação entre qualquer MMP3 } \\
\text { ou o genótipo MMP9 e degeneração. } \\
\text { Sugerido possível papel para o } \\
\text { polimorfismo MMP1 na degeneração da } \\
\text { ATM. }\end{array}$ \\
\hline Smith et al $^{18}$ & 2011 & 1960 & $\begin{array}{l}\text { Americanos } \\
\text { (EUA) }\end{array}$ & $\begin{array}{l}358 \text { genes, dentre eles } \\
\text { HTR2A; COMT }\end{array}$ & $\begin{array}{l}\text { Associação entre o polimorfismo de } \\
\text { HTR2A e COMT; outros genes foram } \\
\text { revelados como possíveis fatores de } \\
\text { risco para a DTM, tais quais NR3C1, } \\
\text { CAMK4, CHRM2, IFRD1, e GRK5 }\end{array}$ \\
\hline
\end{tabular}

Abreviaturas usadas na tabela: 5-HTT: transportador de serotonina-5; 5-HT2A: receptor de serotonina; ADRB2: receptor adrenérgico tipo 2; ERalpha: receptor de estrogênio tipo alfa; COMT: Catecol-O-metiltransferase; MMP: metaloproteinases. Fonte: Elaborada pelos autores 
de quais indivíduos são mais propensos a desenvolver desordens da ATM e/ou na previsão da gravidade desta doença. ${ }^{6} \mathrm{O}$ mecanismo de destruição da ATM é complexo e, como outras doenças complexas, pode envolver polimorfismos genéticos em regiões responsáveis pela organização do RNA (exons) e, por consequência, influenciar na transcrição de proteínas que participam na degeneração da ATM. ${ }^{19}$ Diferentes loci genéticos podem estar associados ao desenvolvimento desta patologia, cada um contribuindo com pequenos efeitos que interagem com exposições ambientais, determinando o curso e o resultado da desordem. ${ }^{20}$ Outros mecanismos que têm sido referidos à DTM são fatores relacionados à percepção psicopatológica e dor, que também podem ser influenciadas pela variabilidade genética. ${ }^{25}$ Muitas vezes, apenas componentes físicos e químicos envolvidos no evento doloroso são considerados; no entanto, aspectos subjetivos e psicológicos, característicos de pacientes com dor crônica, ${ }^{33}$ são cruciais na compreensão da queixa dolorosa.

Na literatura existem inúmeras correlações entre DTM e polimorfismo de genes, tais quais receptor de serotonina, receptor $a$ do estrogênio, Catecol-O-metiltransferase (COMT), vias metabólicas do folato e outras. No entanto, até o momento nenhum estudo correlacionou de forma clara a presença de DTM com genes específicos que regulam a resposta inflamatória no sistema músculoesquelético. Estudos prospectivos que incluam grande número de pacientes e analisem um maior número de moléculas envolvidas na resposta inflamatória são necessários para confirmar a associação entre polimorfismo genético e o desenvolvimento da desordem temporomandibular. No entanto, em estudos em genética, é de extrema importância comparativa distinguir a origem das populações analisadas, as quais podem possuir particularidades nas regiões polimórficas do DNA, e os critérios de diagnóstico de DTM, o qual pode variar abundantemente de um estudo para outro.

Outro fator importante no estudo de genética em populações é a presença de dados epidemiológicos claros e aplicáveis. No Brasil, os dados epidemiológicos relativos aos pacientes portadores de DTM são raros ou inexistentes. No entanto, sabe-se que é alta a frequência de pacientes à procura de tratamento das DTMs em faculdades e clínicas de odontologia. Paradoxalmente, o Brasil é carente de centros que supram as necessidades de atendimento a pacientes portadores de DTM. Isto indica a urgente necessidade de centros de referência com o intuito de diagnosticar, tratar e melhor conhecer a distribuição destas condições em nossa população. ${ }^{32}$

Acredita-se que futuros métodos diagnósticos e terapêuticos poderão ter como base a característica genética do indivíduo, seja ela protetora ou destrutiva, de acordo com a identificação de regiões polimórficas, as quais já vêm sendo alvo da indústria farmacêutica. Esse avanço no entendimento e individualização da etiopatogenia da DTM proporcionará aumento da qualidade de vida dos pacientes, com tratamentos eficazes direcionados para cada caso, especificamente, além de contribuir para a redução de gastos com essa patologia em serviços públicos.

\section{Conclusão}

Baseando-se nos artigos revisados, este estudo demonstra que a característica genética tem correlação com o desenvolvimento de DTM. No entanto, este fator polimórfico precisa ser explorado, considerando a etiologia multifatorial da doença, as características da população estudada e o diagnóstico preciso. Portanto, futuros estudos poderão ser desenvolvidos como forma de melhor entender a etiopatogenia da DTM e suas inúmeras consequências. 


\section{REFERÊNCIAS}

1. Celic R, Jerolimov V, Zlataric DK, Klaic B. Measurement of mandibular movements in patients with temporomandibular disorders and in asymptomatic subjects. Coll Antropol, 2003 Dec; 27(2): 43-9.

2. Shinozaki EB, Paiva G, Zanin FAA, Brugnera Júnior A. Avaliação eletromiográfica de pacientes com DTM após a laserterapia. RGO. 2006 Out-Dez; 54(4): 334-9.

3. Tosato JP, Caria PHF. Prevalência de DTM em diferentes faixas etárias. RGO. 2006 Jul-Set; 54(3): 211-24.

4. Wang X, Guo H, Wang Y, Yi X. The effects of estrogen on cytoplasmic $\mathrm{ca}^{2+}$ concentration of masticatory muscles myoblast in acid condition. 5th International Conference on Bioinformatics and Biomedical Engineering (iCBBE); 10-12 May 2011; Wuhan, China. Anais. Disponível em: http://www.icbbe.org/2011/Proceeding2010. aspx.

5. Torres-Chávez KE, Fischer L, Teixeira JM, Fávaro-Moreira NC, Obando-Pereda GA, Parada CA, Tambeli $\mathrm{CH}$. Sexual dimorphism on cytokines expression in the temporomandibular joint: the role of gonadal steroidal hormones. Inflammation. 2011; 34(5): 487-98.

6. Wadhwa S, Kapila S. TMJ Disorders: future innovations in diagnostics and therapeutics. J Dent Educ. 2008; 72(8): 930-47.

7. Coutinho MEP, Wassall T, Ferrer KJN, Magalhães JCA. Os efeitos do tratamento ortodôntico sobre a articulação temporomandibular. RGO. 2003 Out; 51(4): 335-42.

8. Alves BM, Macedo CR, Januzzi E, Grossmann E, Atallah AN, Peccin S. Mandibular manipulation for the treatment of temporomandibular disorder. J Craniofac Surg. 2013 Mar; 24(2): 488-93.

9. Almăşan $O C$, Băciuţ $M$, Almăşan $H A$, Bran $S$, Lascu L, lancu M, Băciuţ G. Skeletal pattern in subjects with temporomandibular joint disorders. Arch Med Sci. 2013; 9(1): 118-26.

10. Meloto CB, Serrano PO, Ribeiro Dasilva MCR, Rizzatti-Barbosa C. Genomics and the new perspectives for temporomandibular disorders. Arch Oral Biol. 2011; 56: 1181-91.

11. Denadai-Souza A, Cenac N, Casatti CA, Souza Câmara PR, Yshii LM, Costa SKP et al. PAR ${ }_{2}$ and temporomandibular joint inflammation in the rat. J Dent Res. 2010; 89(10): 1123-28.

12. Rauhala K., Oikarinen KS, Raustia AM. Role of temporomandibular disorders (TMD) in facial pain: occlusion, muscle and TMJ pain. Cranio. 1999 Oct; 17(4): 254-61.

13. Manfredini D, Favero L, Gregorini G, Cocilovo F, Guarda-Nardini L. Natural course of temporomandibular disorders with low painrelated impairment: a 2-to-3-year follow-up study. J Oral Rehabil. 2013 Mar 22; 40(6): 43642.

14. Tanaka E, Detamore MS, Mercuri LG. Degenerative disorders of the temporomandibular joint: etiology, diagnosis, and treatment. J Dent Res. 2008; 87(4): 296307.

15. Lopes PRR, Campos PSF, Nascimento RJN. Dor e inflamação nas disfunções temporomandibulares: revisão de literatura dos últimos quatro anos. Rev Cie. Med Miol. 2011 Set-Dez; 10(3): 317-25.

16. Thompson MW, Mcinnes RR, Willard HF. Genes in Population. In: Nussbaum RL, McInnes RR, Willard HF, editors. Thompson and Thompson Genetics in medicine. 6th ed. Philadelphia, PA: WB Saunders Company; 2001. p. 540.

17. Zitzmann NU, Berglundh T. Definition and prevalence of peri-implant disease. J Clin Periodontol. 2008; 35: 286-91.

18. Smith SB, Maixner DW, Greenspan JD, Dubner $\mathrm{R}$, Fillingim RB, Ohrbach $\mathrm{R}$ et al. Potential genetic risk factors for chronic TMD: genetic associations from the OPPERA case control study. The Journal of Pain. 2011 Nov; 12(11): T92-T101.

19. Planello AC, Campos MIG, Meloto CB, Secolin $\mathrm{R}$, Rizatti-Barbosa CM, Line SRP et al. Association of matrix metalloproteinase gene polymorphism with temporomandibular joint degeneration. Eur J Oral Sci. 2011; 119: 1-6.

20. Diatchenko L, Anderson A, Slade G, Fillingim R, Shabalina S, Higgins $T$ et al. Three major haplotypes of the B2 adrenergic receptor define psychological profile, blood pressure, and the risk for development of a common musculoskeletal pain disorder. Am J Med Genet. 2006; 141B(5): 449-62.

21. Huang B, Takahashi K, Sakata T, Kiso H, Sugai $M$, Fujimura $K$ et al. Increased risk of temporomandibular joint closed lock: a casecontrol study of ANKH polymorphisms. Plos One. 2011 Oct; 6(10): 1-7.

22. Herken $H$, Erdal E, Mutlu N, Barlas $O$, Cataloluk O, Oz F. Possible association of temporomandibular joint pain and dysfunction 
with a polymorphism in the serotonin transporter gene. Am J Orthod Dentofacial Orthop. 2011 Sep; 120(3): 308-13.

23. Mutlu N, Erdal ME, Herken H, Oz G, Bavazit YA. T102C polymorphism of the 5-HT2A receptor gene may be associated with temporomandibular dysfunction. Oral Dis. 2004 Nov; 10(6): 349-52.

24. Kang SC, Lee DG, Choi JH, Kim ST, Kim YK, Ahn HJ. Association between estrogen receptor polymorphism and pain susceptibility in female temporomandibular joint osteoarthritis patients. Int J Oral Maxillofac Surg. 2007 May; 36(5): 391-4.

25. Aneiros-Guerrero A, Lendinez AM, Palomares AR, Perez-Nevot B, Aguado L, Mayor-Olea A et al. Genetic polymorphisms in folate pathway enzymes, DRD4 and GSTM1 are related to temporomandibular disorder. BMC Medical Genetics. 2011; 12: 75.

26. Ojima K., Watanabe N, Narita N, Narita M. Temporomandibular disorder is associated with a serotonin transporter gene polymorphism in the Japanese population. BioPsychoSocial. 2007; 1(3): 1-4.

27. Slade GD, Diarchenko L, Bhalang K, Sigurdsson A, Fillingim RB, Belfer $\mathrm{I}$ et al. Influence of psychological factors on risk of temporomandibular disorders. J Dent Res. 2007 Nov; 86(11): 1120-25.

28. Kim BS, Kim YK, Yun PY, Lee E, Bae J. The effects of estrogen receptor a polymorphism on the prevalence of symptomatic temporomandibular disorders. J Oral Maxillofac Surg. 2010 Dec; 68(12): 2975-79.
29. Tchivileva IE, Lim PF, Smith SB, Slade GD, Diatchenko L, McLean Sa et al. Effect of catechol-O-methyltransferase polymorphism on response to propranolol therapy in chronic musculoskeletal pain: a randomized, doubleblind, placebo-controlled, crossover pilot study. Pharmacogenet Genomics. 2010 Apr; 20(4): 239-48.

30. Durham J. Temporomandibular disorders (TMD): an overview. Oral Surgery. 2008; 1(2): 60-8.

31. Demarim V, Kes VB. Temporomandibular disorders and migraine headache. Medical Sciences. 2010; 34: 111-7.

32. Pereira Júnior FJ, Vieira AR, Miasato JM. Visão geral das Desordens Temporomandibulares. Parte I: definição, epidemiologia e etiologia. RGO. 2004; 52(2): 117-21.

33. Oliveira AS, Bermudez CC, Souza RA, Souza CMF, Dias EM, Castro CES et al. Impacto da dor na vida de portadores de disfunção temporomandibular. J Appl Oral Sci. 2003; 11(2): 138-43.

34. Cordeiro IB, Guimarães AS. Profile of patients with temporomandibular joint disorder: main complaint, signs, symptoms, gender and age. RGO. 2012 Abr-Jun; 60(2): 143-8.

\section{Autor correspondente:}

Priscila Ladeira Casado:

Endereço: Rua Marques de Paraná 303 - 40 andar -

Centro - Niterói -

Rio de Janeiro - Brasil - 24033-900

Submetido em: 7-1-2013

Aceito em: 19-8-2013 